Supporting Information

\title{
Fluorescent Polystyrene Microbeads as Invisible Security Ink and Optical Vapor Sensor for 4-Nitrotoluene
}

\author{
Swapnil L. Sonawane ${ }^{\text {a,b }}$ and S. K. Asha*a,b,c
}

a) Polymer Science and Engineering Division, CSIR-National Chemical Laboratory, Dr Homi Bhabha Road, Pune 411008, India.

b) Academy of Scientific and Innovative Research, New Delhi, India

c) CSIR-Network Institutes of Solar Energy, New Delhi, India Corresponding author e-mail address: $\underline{\text { sk.asha@ncl.res.in }}$ 
Table S1. Dispersion Polymerization contents

Table S2. Fluorescence Lifetime Decay Fit Parameters. The decay time collected at $395 \mathrm{~nm}$ by using nano LED $340 \mathrm{~nm}$ for excitation of pyrene.

Table S3. Fluorescence Lifetime Decay Fit Parameters. The decay time collected at $530 \mathrm{~nm}$ by using nano LED $460 \mathrm{~nm}$ for excitation of PBI.

Figure S1a. ${ }^{1} \mathrm{H}$ NMR spectrum of Pyrene-Acrylate recorded in $\mathrm{CDCl}_{3}$. b. MALDI-TOF spectrum of Pyrene-Acrylate.

Figure S2. Comparative ${ }^{1} \mathrm{H}$ NMR spectra of PS-Py-DEG-4 and Pyrene-Acrylate recorded in $\mathrm{CDCl}_{3}$.

Figure S3a. The size exclusion chromatography of all PS-Py-PBI polymers. b. Thermo gravimetric Analysis (TGA) of all PS-Py-PBI samples.

Figure S4a. Volume - average size distribution of PS-Py-PBI samples in ethanol dispersion obtained by dynamic light scattering (DLS) analysis. b. SEM images $(1 \mathrm{mg} / 2 \mathrm{~mL}$ ethanol dispersion) sample. c. Fluorescence optical microscopy images using DAPI (350-450 nm Blue), Alexa (488-520 Green) and Rhodamine (480-580 nm Red) filters and (c) PS-Py-DEG-4 using DAPI (350-450 nm Blue) (d) PS-PBI-5 using Alexa (488-520 Green) and Rhodamine (480-580 nm Red).

Figure S5. Normalized absorption and Emission spectra of Pyrene-Acrylate and PBI in $\mathrm{CHCl}_{3}$ respectively. (0.1 OD at $343 \mathrm{~nm} ; \lambda_{\mathrm{ex}}=343 \mathrm{~nm}$ for Pyrene-Acrylate and $0.1 \mathrm{OD}$ at $527 \mathrm{~nm} ; \lambda_{\mathrm{ex}}=$ $490 \mathrm{~nm}$ for $\mathbf{P B I})$.

Figure S6. Fluorescence decay profiles (LED 340 nm, em: $397 \mathrm{~nm}$ and LED 460 nm, em: 530 nm) of Pyrene-Acrylate, PS-Py-DEG-4, PS-Py-PBI-1, PS-Py-PBI-3 and PS-PBI-5 in the powder state.

Figure S7. Time dependant fluorescence quenching of PS-Py-PBI-1 (excitation at $343 \mathrm{~nm}$ for Pyrene and excitation at $490 \mathrm{~nm}$ for PBI) in (a, b) powder form, spincoated films from (c, d) ethanol dispersion and (e, f) chloroform upon exposure to 4-nitrotoluene vapors.

Figure S8. Excitation spectra of PS-Py-PBI-1 (excitation at $415 \mathrm{~nm}$ for Pyrene and $576 \mathrm{~nm}$ for PBI respectively) in (a) powder form, spincoated from (b) ethanol dispersion and (c) chloroform upon exposure to 4-nitrotoluene vapors for 60 minutes. 


\section{Synthesis of Pyrene Acrylate ${ }^{1}$}

The synthesis of pyrene acrylate In a $100 \mathrm{~mL}$ round-bottom flask, pyrene methanol (200 mg, $0.00085 \mathrm{~mol})$ and $\mathrm{Et}_{3} \mathrm{~N}(0.77 \mathrm{~mL}, 0.0055$ mole) along with catalytic amount of DMAP (dimethyl amino pyridine) was dissolved in dry THF $(17 \mathrm{~mL})$ under nitrogen atmosphere and stirred well at $0^{\circ} \mathrm{C}$ for 15 minute. Acryloyl chloride $(0.19 \mathrm{~mL}, 0.0023 \mathrm{~mol})$ was taken in THF $(3 \mathrm{~mL})$ and added to the reaction mixture for a period of $15-20 \mathrm{~min}$ at $0^{\circ} \mathrm{C}$. The reaction was carried out for 4 hour at room temperature and monitored by TLC. The organic layer was evaporated and the compound was purified by column chromatography in $20 \%$ ethyl acetate/pet ether solvent combination. Yield $=185 \mathrm{mg}(78 \%) .{ }^{1} \mathrm{H}$ NMR (200 MHz, $\left.\mathrm{CDCl}_{3}, \delta \mathrm{ppm}\right): 8.19-8.08$ (m, 9H, Ar-H) 6.43 (dd, 2H, acrylic double bond), 6.19 (m, 2H, acrylic double bond), 5.87 (dd, 2H, acrylic double bond), 5.92 (s, $\left.2 \mathrm{H}, \mathrm{Ar}-\mathrm{CH}_{2}-\mathrm{O}\right) .{ }^{13} \mathrm{C} \mathrm{NMR}\left(200 \mathrm{MHz}, \mathrm{CDCl}_{3}\right)$ 166.3, 131.7, 131.1, $130.1,130.6$, 129.5, 128.6, 128.2, 127.8, 127.7, 127.3, 126, 125.5, 125.4, 124.6, 122.8, 64.9. MALDI-TOF-MS (Dithranol matrix): m/z calcd for $\mathrm{C}_{20} \mathrm{H}_{14} \mathrm{O}_{2}: 286.30$; found 286.15 [M ${ }^{+}$]

Table S1. Dispersion Polymerization contents

\begin{tabular}{|c|c|c|c|c|}
\hline Component & Material & $\begin{array}{c}\text { Amount } \\
(\mathbf{g m})\end{array}$ & $\begin{array}{c}\text { Amount } \\
\mathbf{( g m )}\end{array}$ & $\begin{array}{c}\text { Amount } \\
\mathbf{( g m )}\end{array}$ \\
\hline Monomer & Styrene & 1.65 & 1.43 & $3^{\text {rd }}$ Stage \\
\hline Co-monomer & Pyrene Acrylate & No & 0.001, to 0.100 & 0.666 \\
\hline Cross-linker & PBI/DEG & No & - & 0.0024 to $0.0032 \mathrm{gm} / 0.001$ \\
\hline Medium & Ethanol & 7.4 & 5.59 & 1.86 \\
\hline Stabilizer & PVP $(360,000)$ & 0.25 & No & No \\
\hline Co-Stabilizer & Triton X-100 & 0.08 & No & No \\
\hline Initiator & AIBN & 0.060 & No & - \\
\hline Reaction time & 8 hours & - & - & - \\
\hline Rotation Speed & $120 \mathrm{rpm}$ & - & - & No \\
\hline
\end{tabular}




\section{Dispersion Polymerization procedure for the preparation of PS-Py-PBI-x}

Procedure: The stabilizer PVP (polyvinylpyrrolidone-360,000) (0.25 gm), the co-stabilizer (Triton X-305) (0.086 gm), initiator (AIBN, $0.060 \mathrm{gm})$, styrene monomer (1.65 gm) and ethanol (7.4 gm) were added to a $100 \mathrm{~mL}$ three necked reaction flask equipped with a gas inlet, overhead stirrer and rubber septum. After a homogeneous solution formed at room temperature, the solution was deoxygenated by bubbling nitrogen gas at room temperature for at least 30 minutes. Then the flask was placed in a $70{ }^{\circ} \mathrm{C}$ oil bath and stirred mechanically at $120 \mathrm{rpm}$. The pyrene acrylate was dissolved in styrene (1.43 gm) and ethanol (5.59 gm) and, added to the reaction mixture. In the third stage the cross-linker (PBI) was dissolved in the Styrene (0.666 gm) and ethanol $(1.86 \mathrm{gm})$ at $60{ }^{\circ} \mathrm{C}$ under nitrogen. After the cross-linker had dissolved and the polymerization reaction had run for 15 minute, the hot Styrene-cross-linker-ethanol solution was added into the reaction flask over a period of one hour (drop by drop addition) and polymerization was continued for 6 hours under continuous flow of nitrogen with overhead stirring. The precipitated polymer in the reaction medium was washed with $200 \mathrm{~mL}$ x 4 times of methanol and separated by centrifuge. The polymer was dried under vacuum at $50{ }^{\circ} \mathrm{C}$ for 6 hours. 
Table S2. Parameters ( $\tau$ : Decay Time, $\alpha$ : Pre-exponential Factor, $\chi 2$ : Chi-Squared Value) retrieved from the triexponential fit for samples in powder form. The decay time was collected at $395 \mathrm{~nm}$ by using nano LED $340 \mathrm{~nm}$ for excitation of pyrene.

\begin{tabular}{|c|c|c|c|c|c|}
\hline Sample & $\begin{array}{c}\boldsymbol{\tau}_{\boldsymbol{1}} \\
\left(\boldsymbol{\alpha}_{\mathbf{1}}\right)\end{array}$ & $\begin{array}{c}\boldsymbol{\tau}_{\mathbf{2}} \\
\left(\boldsymbol{\alpha}_{\mathbf{2}}\right)\end{array}$ & $\begin{array}{c}\boldsymbol{\tau}_{3} \\
\left(\boldsymbol{\alpha}_{\mathbf{3}}\right)\end{array}$ & $\begin{array}{c}\mathbf{C h i}^{2} \\
\chi^{2}\end{array}$ & Av. $\boldsymbol{\tau}$ (sec) \\
\hline Pyrn-Acrylate & $\begin{array}{c}2.8 \times 10^{-9} \\
(0.03)\end{array}$ & $\begin{array}{c}6.17 \times 10^{-11} \\
(0.97)\end{array}$ & $\begin{array}{c}1.2 \times 10^{-8} \\
(0.01)\end{array}$ & 1.04 & $2.19 \times 10^{-10}$ \\
\hline PS-Py-DEG-4 & $\begin{array}{c}1.96 \times 10^{-8} \\
(0.00)\end{array}$ & $\begin{array}{c}3.92 \times 10^{-11} \\
(0.99)\end{array}$ & $\begin{array}{c}1.69 \times 10^{-7} \\
(0.01)\end{array}$ & 0.97 & $2.21 \times 10^{-9}$ \\
\hline PS-Py-PBI-1 & $\begin{array}{c}7.7 \times 10^{-8} \\
(0.00)\end{array}$ & $\begin{array}{c}9.5 \times 10^{-11} \\
(0.98)\end{array}$ & $\begin{array}{c}1.57 \times 10^{-7} \\
(0.02)\end{array}$ & 1.02 & $2.75 \times 10^{-9}$ \\
\hline PS-Py-PBI-3 & $\begin{array}{c}1.54 \times 10^{-8} \\
(0.00)\end{array}$ & $\begin{array}{c}4.33 \times 10^{-11} \\
(0.99)\end{array}$ & $\begin{array}{c}1.60 \times 10^{-7} \\
(0.01)\end{array}$ & 0.93 & $2.26 \times 10^{-9}$ \\
\hline
\end{tabular}

Table S3. Parameters ( $\tau$ : Decay Time, $\alpha$ : Pre-exponential Factor, $\chi 2$ : Chi-Squared Value) retrieved from the triexponential fit for samples in powder form. The decay time was collected at $530 \mathrm{~nm}$ by using nano LED $460 \mathrm{~nm}$ for excitation of PBI.

\begin{tabular}{|c|c|c|c|c|c|}
\hline Sample & $\begin{array}{c}\boldsymbol{\tau}_{\mathbf{1}} \\
\left(\boldsymbol{\alpha}_{\mathbf{1}}\right)\end{array}$ & $\begin{array}{c}\boldsymbol{\tau}_{\mathbf{2}} \\
\left(\boldsymbol{\alpha}_{\mathbf{2}}\right)\end{array}$ & $\begin{array}{c}\boldsymbol{\tau}_{3} \\
\left(\boldsymbol{\alpha}_{\mathbf{3}}\right)\end{array}$ & $\begin{array}{c}\mathbf{C h h i}^{\mathbf{2}} \\
\chi^{2}\end{array}$ & $\mathbf{A v .} \boldsymbol{\tau}$ (sec) \\
\hline PS-PBI-5 & $\begin{array}{c}2.7 \times 10^{-9} \\
(0.04)\end{array}$ & $\begin{array}{c}4.46 \times 10^{-9} \\
(0.71)\end{array}$ & $\begin{array}{c}2.8 \times 10^{-10} \\
(0.25)\end{array}$ & 1.21 & $3.34 \times 10^{-9}$ \\
\hline PS-Py-PBI-1 & $\begin{array}{c}2.15 \times 10^{-9} \\
(0.03)\end{array}$ & $\begin{array}{c}4.52 \times 10^{-9} \\
(0.81)\end{array}$ & $\begin{array}{c}4.4 \times 10^{-10} \\
(0.16)\end{array}$ & 0.98 & $3.8 \times 10^{-9}$ \\
\hline PS-Py-PBI-3 & $\begin{array}{c}2.93 \times 10^{-9} \\
(0.10)\end{array}$ & $\begin{array}{c}4.49 \times 10^{-9} \\
(0.72)\end{array}$ & $\begin{array}{c}5.6 \times 10^{-10} \\
(0.18)\end{array}$ & 1.12 & $3.64 \times 10^{-9}$ \\
\hline
\end{tabular}




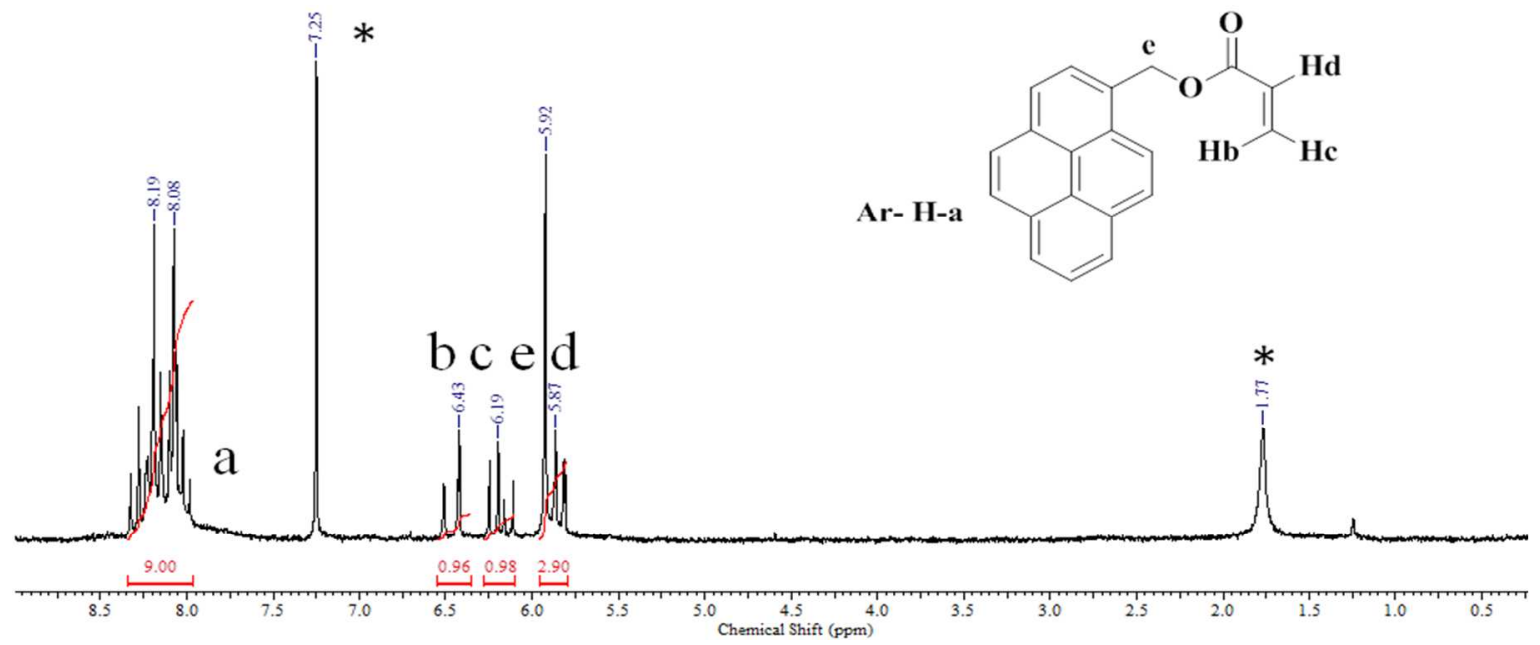

Figure S1a. ${ }^{1} \mathrm{H}$ NMR spectrum of Pyrene-Acrylate recorded in $\mathrm{CDCl}_{3}$.

Calculated Mass, 286.09

Observed Mass:-286.15, 287.16 (M+1, M+2)

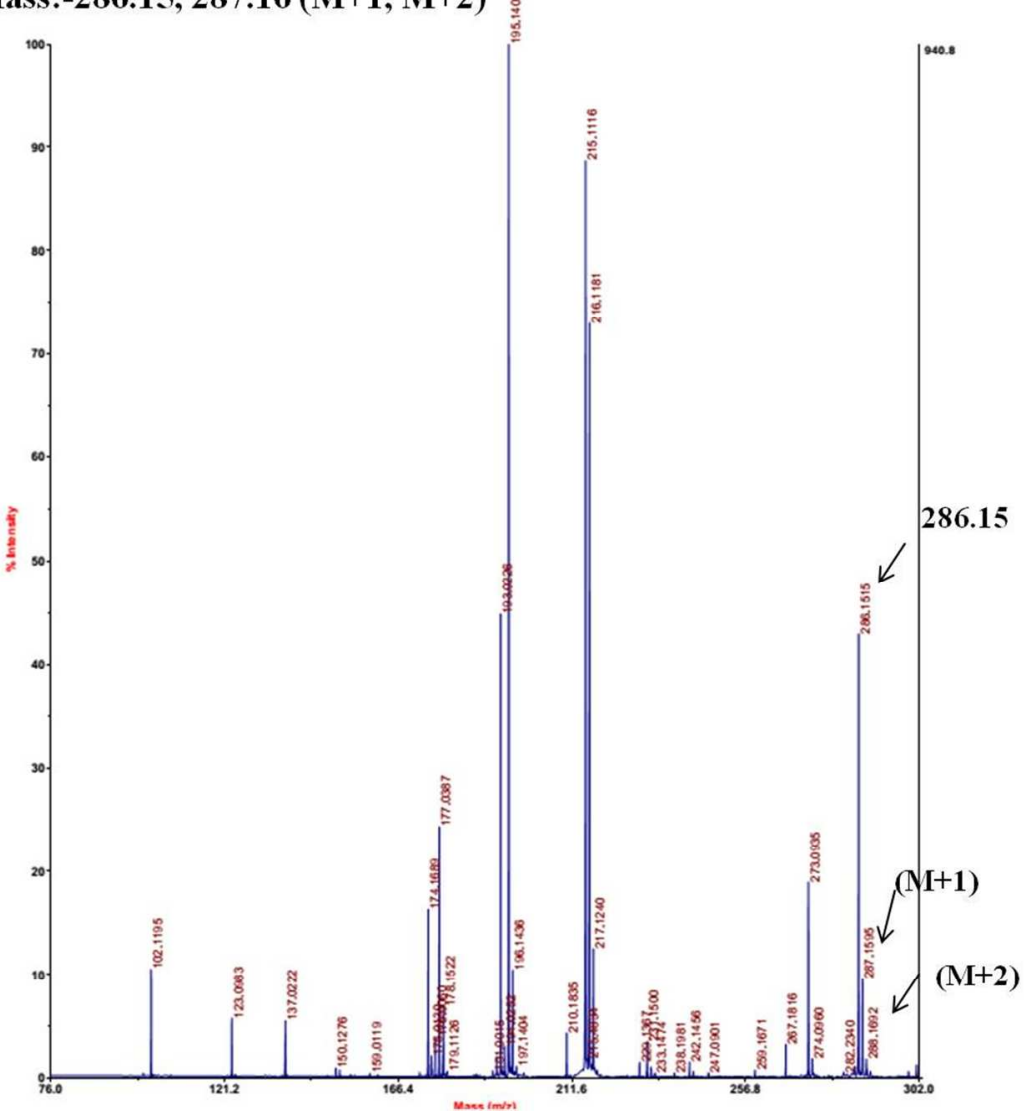

Figure S1b. MALDI-TOF spectrum of Pyrene-Acrylate. 


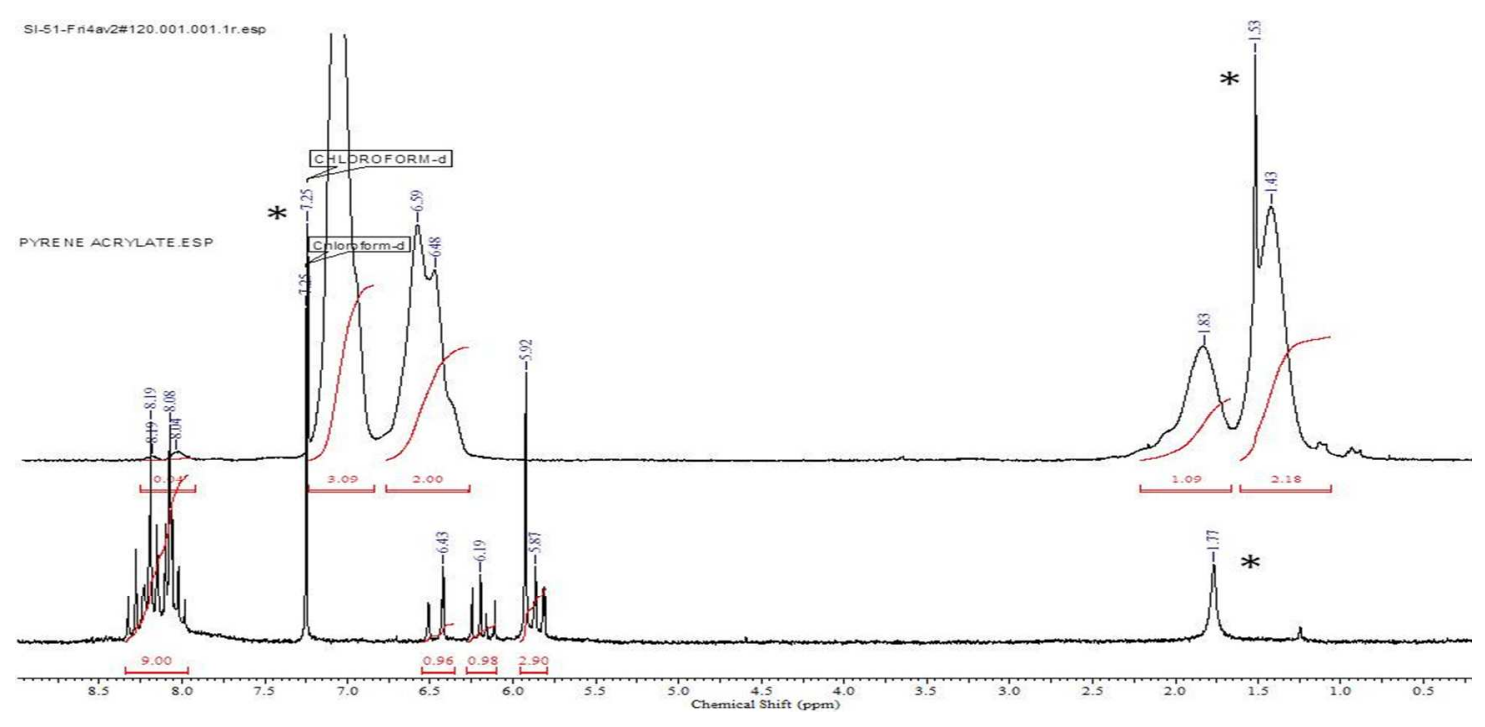

Figure S2. Comparative ${ }^{1} \mathrm{H}$ NMR spectra of PS-Py-DEG-4 and Pyrene-Acrylate recorded in $\mathrm{CDCl}_{3}$.

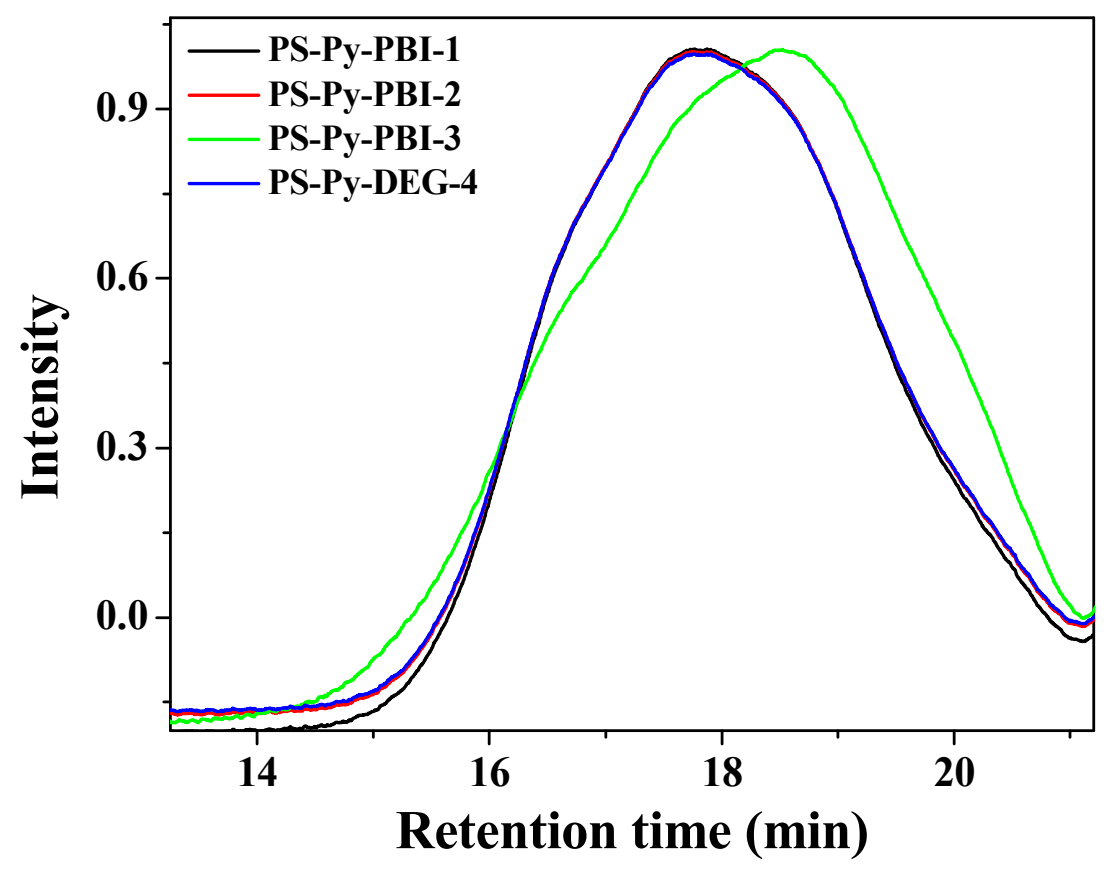

Figure S3a. The size exclusion chromatography of all PS-Py-PBI polymers. 


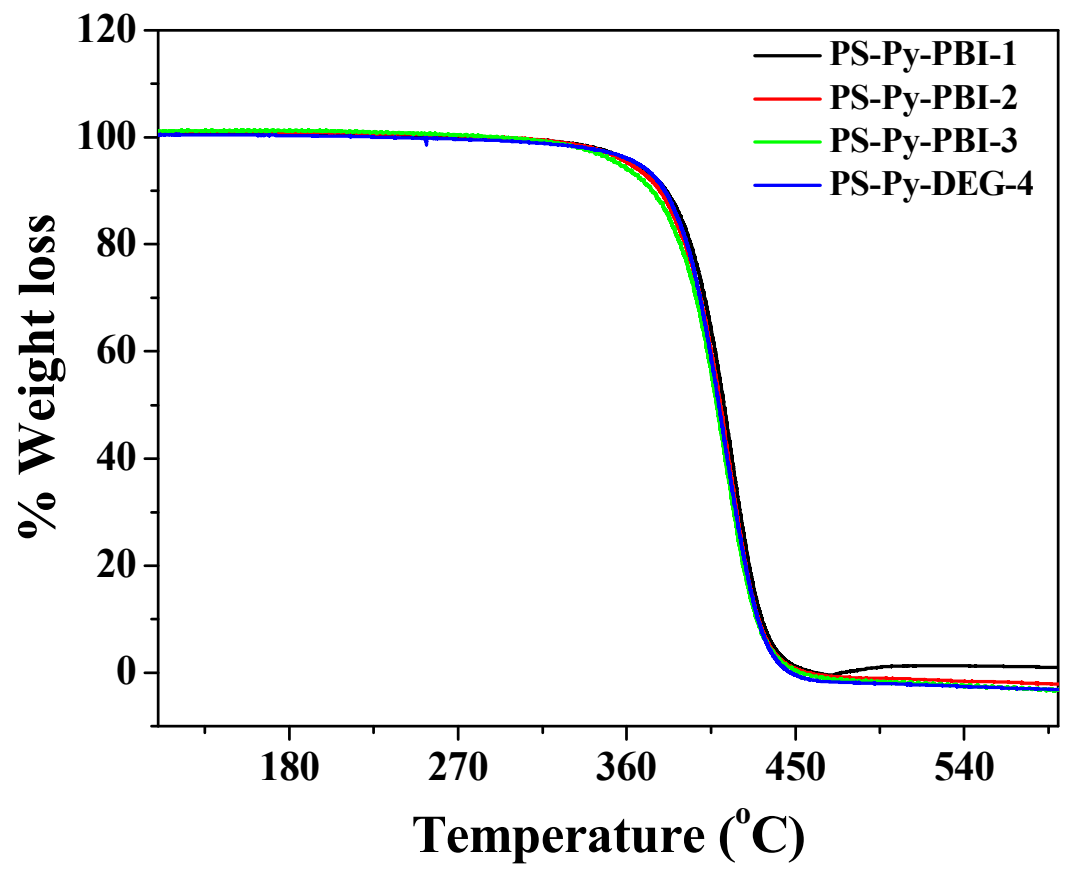

Figure S3b. Thermo gravimetric Analysis (TGA) of all PS-Py-PBI samples.

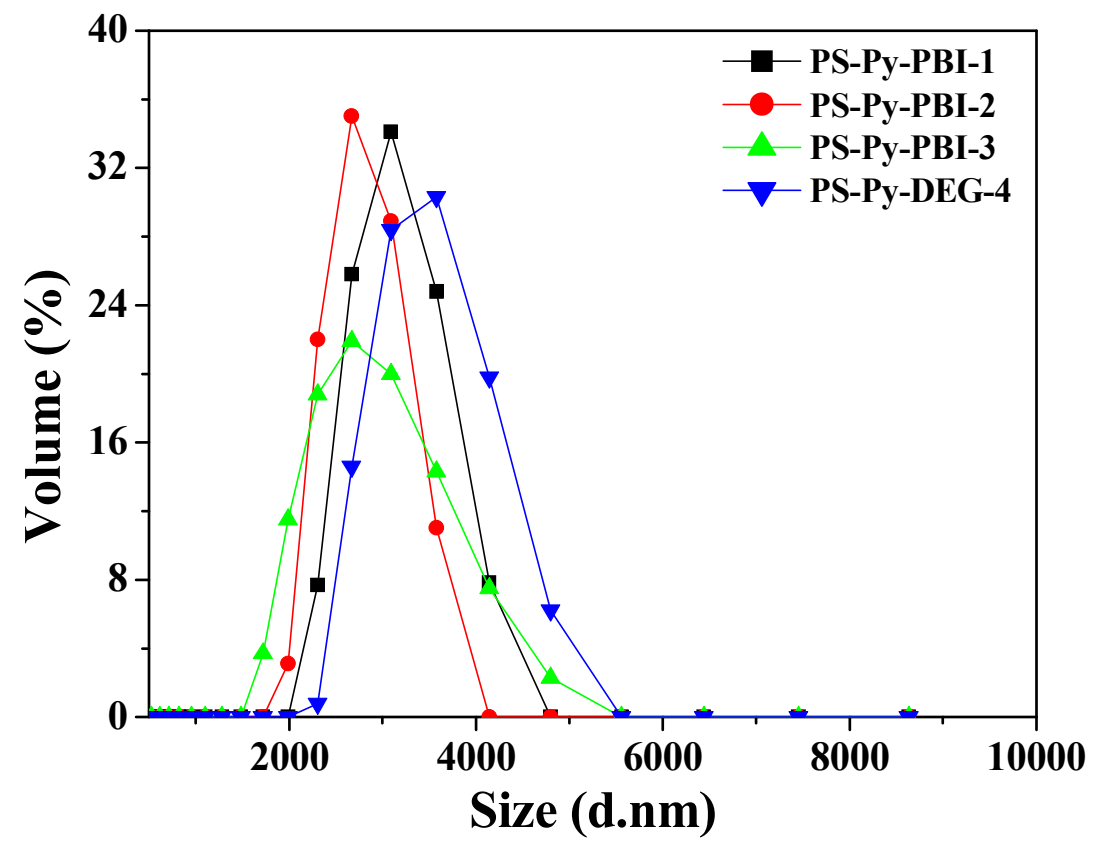

Figure S4a. Volume - average size distribution of PS-Py-PBI samples in ethanol dispersion obtained by dynamic light scattering (DLS) analysis. 


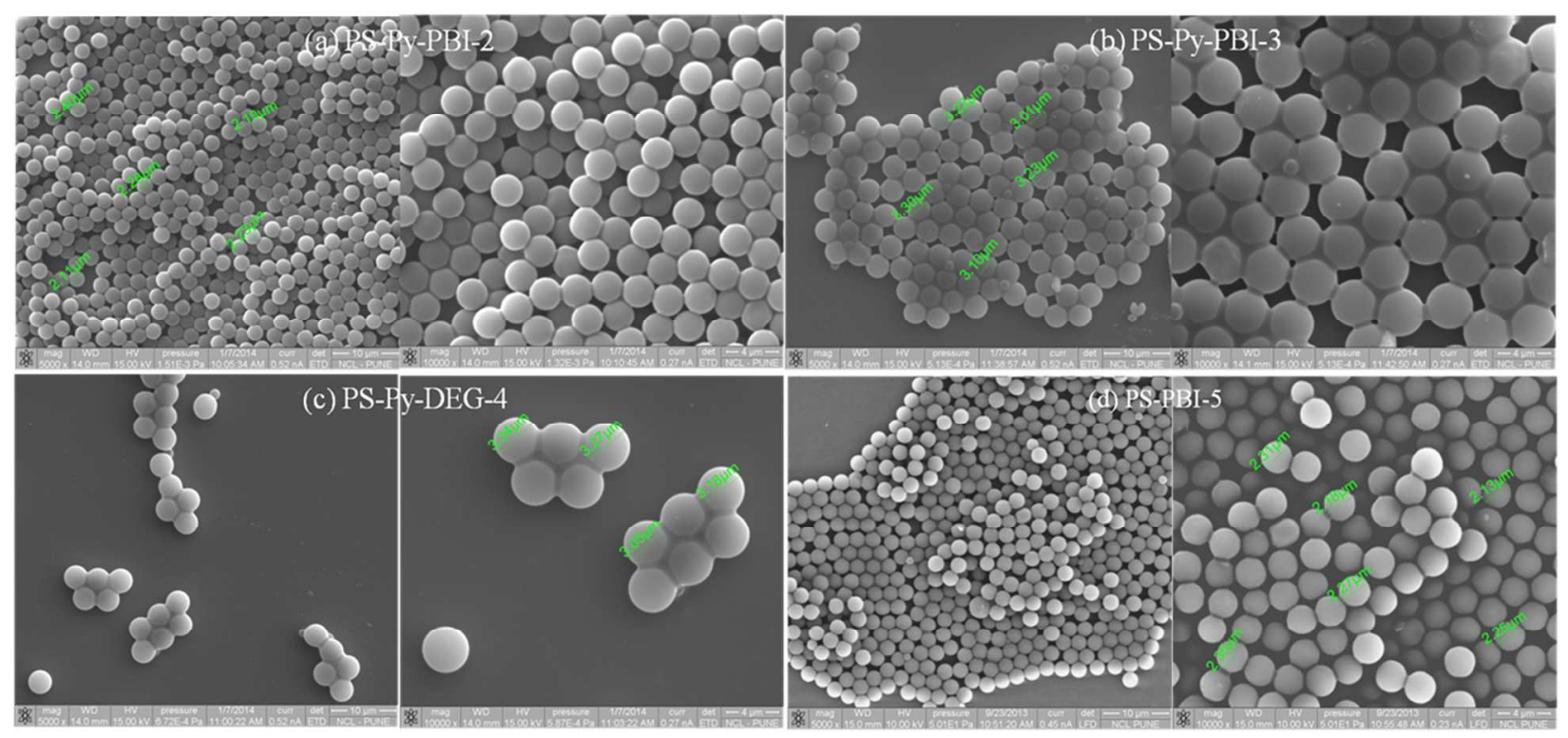

Figure S4b. SEM images of (a) PS-Py-PBI-2 (b) PS-Py-PBI -3 (c) PS-Py-DEG-4 and (d) PSPBI-5 drop cast on silicon wafer ( $1 \mathrm{mg} / 2 \mathrm{~mL}$ ethanol dispersion). 


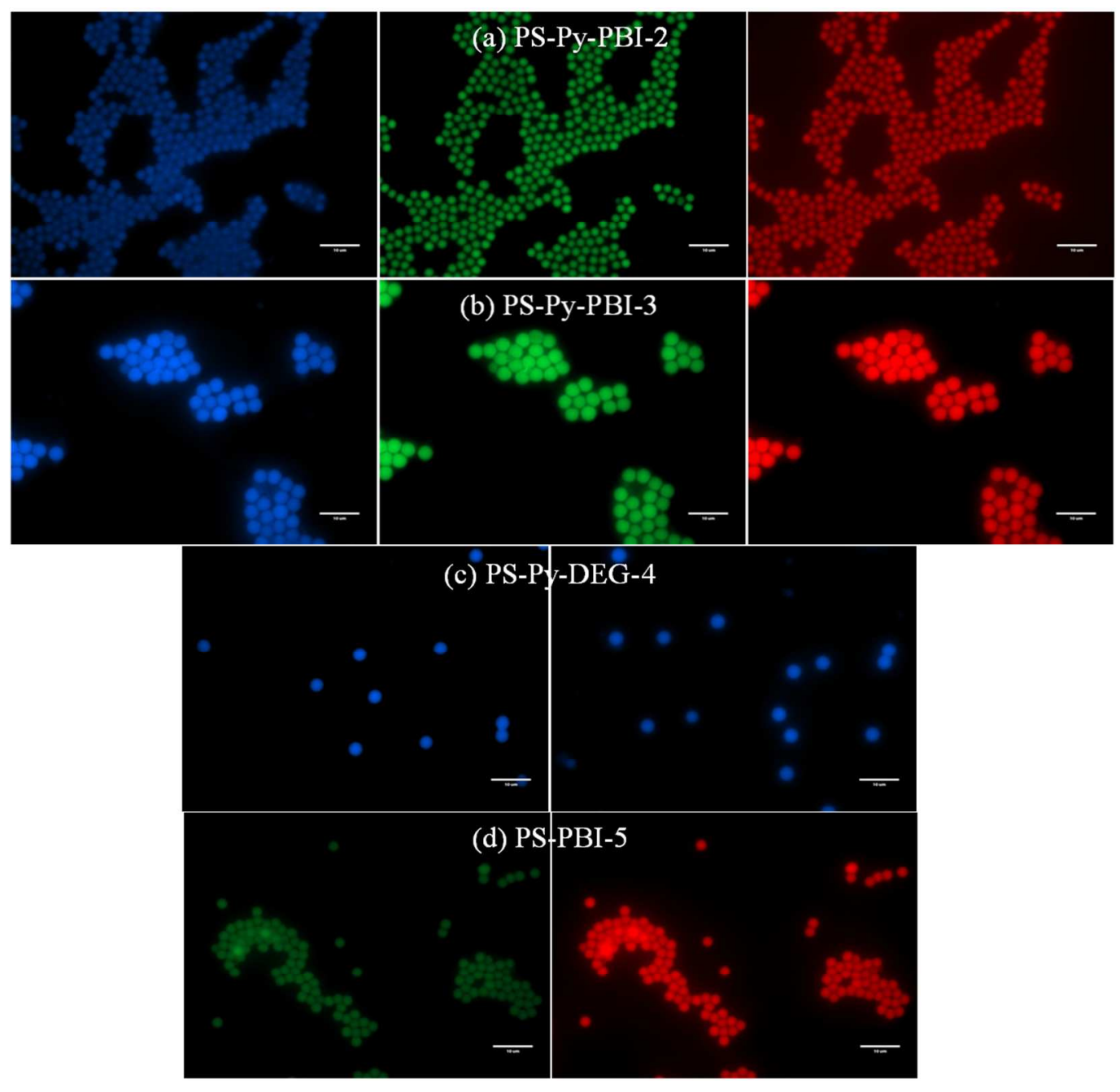

Figure S4c. Fluorescence optical microscopy images of (a) PS-Py-PBI-2 (b) PS-Py-PBI-3 using DAPI (350-450 nm Blue), Alexa (488-520 Green) and Rhodamine (480-580 nm Red) filters and (c) PS-Py-DEG-4 using DAPI (350-450 nm Blue) (d) PS-PBI-5 using Alexa (488-520 Green) and Rhodamine (480-580 nm Red). 


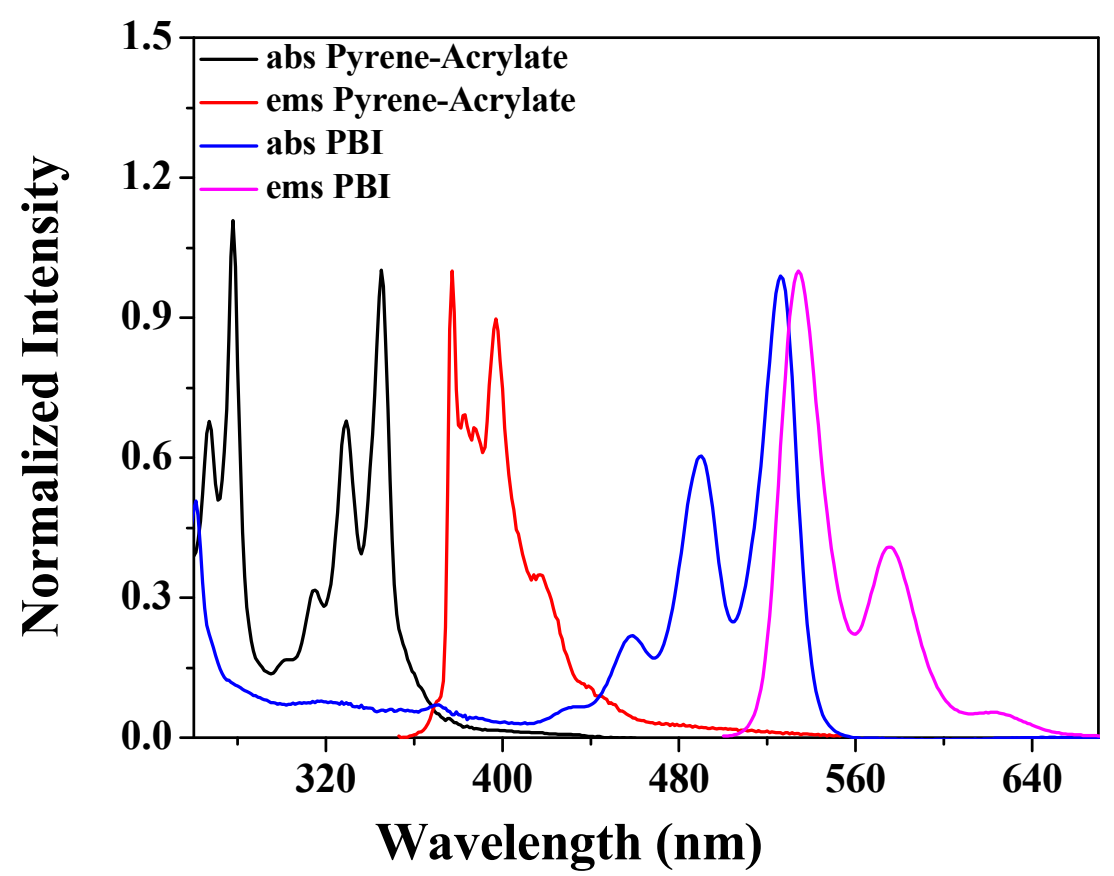

Figure S5. Normalized absorption and Emission spectra of Pyrene-Acrylate and PBI respectively in $\mathrm{CHCl}_{3}$. (0.1 OD at $343 \mathrm{~nm} ; \lambda_{\mathrm{ex}}=343 \mathrm{~nm}$ for Pyrene-Acrylate and $0.1 \mathrm{OD}$ at 527 $\mathrm{nm} ; \lambda_{\text {ex }}=490 \mathrm{~nm}$ for $\left.\mathbf{P B I}\right)$. 


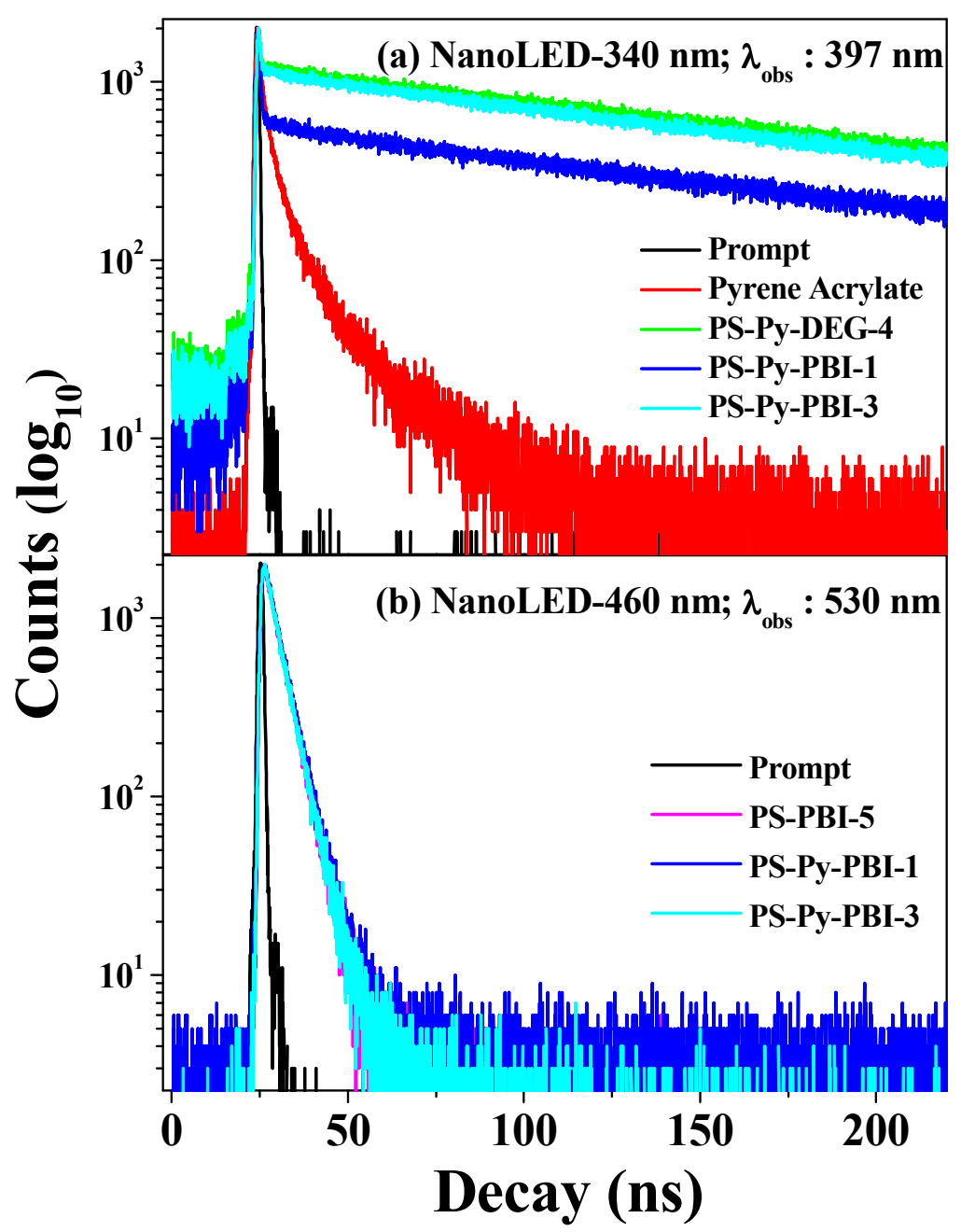

Figure S6. Fluorescence decay profiles (LED 340 nm, em: 397 nm and LED 460 nm, em: 530 nm) of Pyrene-Acrylate, PS-Py-DEG-4, PS-Py-PBI-1, PS-Py-PBI-3 and PS-PBI-5 in the powder state. 


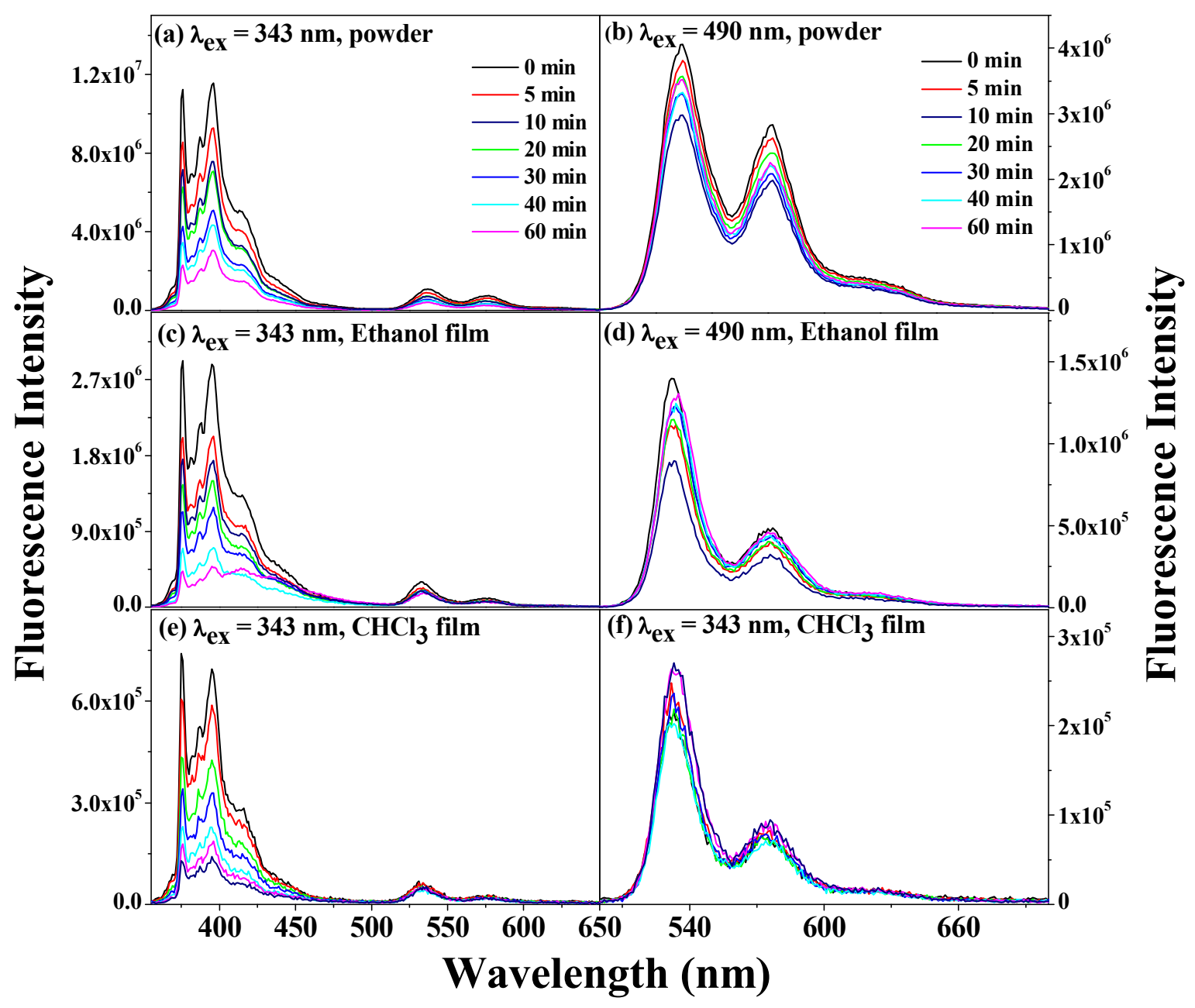

Figure S7. Time dependant fluorescence quenching of PS-Py-PBI-1 (excitation at $343 \mathrm{~nm}$ for Pyrene and excitation at $490 \mathrm{~nm}$ for PBI) in ( $a, b)$ powder form, spincoated films from (c, d) ethanol dispersion and (e, f) chloroform upon exposure to 4-nitrotoluene vapors. 


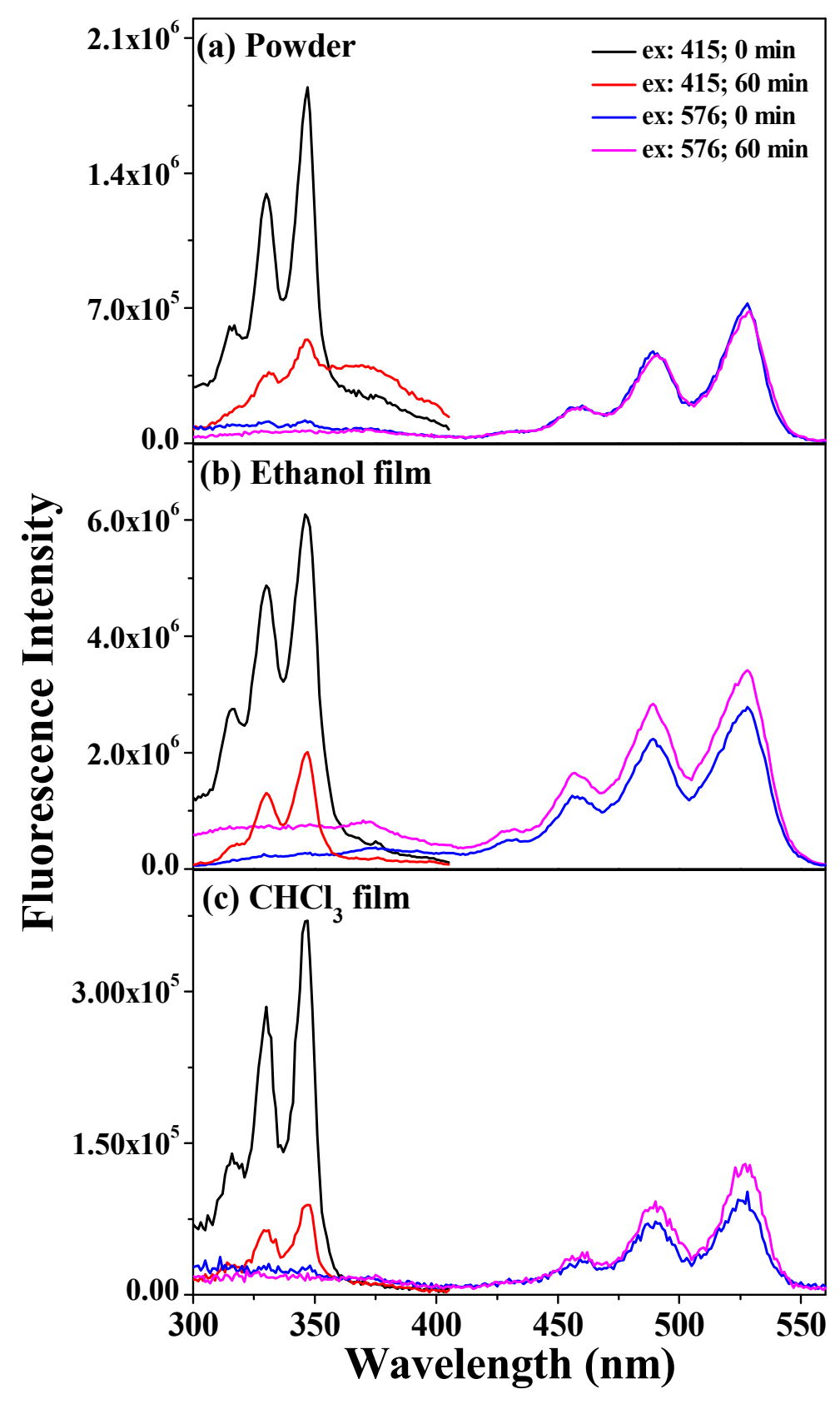

Figure S8. Excitation spectra of PS-Py-PBI-1 (excitation at $415 \mathrm{~nm}$ for Pyrene and $576 \mathrm{~nm}$ for PBI respectively) in (a) powder form, spincoated from (b) ethanol dispersion and (c) chloroform upon exposure to 4-nitrotoluene vapors for 60 minutes.

\section{Reference}

1. Oh, J. K.; Wu, J.; Winnik, M. A.; Craun, G. P.; Rademacher, J.; Farwaha, R. J. Polym. Sci. Polym. Chem. 2002, 40, 1594-1607. 